\title{
Extracting an arbitrary relative phase from a multiqubit two-component entangled state
}

\author{
Chui-Ping Yang and Siyuan Han \\ Department of Physics and Astronomy, University of Kansas, Lawrence,
} Kansas 66045

\begin{abstract}
We show that an arbitrary relative phase can be extracted from a multiqubit two-component (MTC) entangled state by local Hadamard transformations and measurements along a single basis only. In addition, how to distinguish a MTC entangled state with an arbitrary entanglement degree and relative phase from a class of multiqubit mixed states is discussed.
\end{abstract}

PACS numbers: 03.67.Lx, 03.65.Ud, 85.25.Dq

Entanglement plays an important role in quantum information processing and communication, such as quantum computation, secret sharing, teleportation, and quantum key distribution [1-3]. Over the last decade, characterizing or/and quantifying entanglement has been recognized as one of central tasks in quantum information science. Correspondingly, there have been many efforts in this direction, for both pure and mixed states as well as for bipartite and multipartite systems. These include entanglement of formation [4], entanglement of distillation [5], relative entropy of entanglement [6], negativity [7], geometric measure of entanglement [8], and so on.

On the other hand, there is a strong current interest in the detection of quantum entanglement. To verify whether a qubit system is indeed prepared in a desired entangled state, many methods for the entanglement detection have been presented. Among them, there are proposals based on the structural approximations [9], the semidefinite program [10], and the entanglement witnesses [11-14]. In addition, the idea of inferring quantum entanglement using a single measurement basis has been proposed by Schelpe et. al. recently [15].

Quantum states can encode information (e.g., by amplitude, phase, or both) that may only be extracted by analyzing the states as a whole. Stimulated by the rapid development in quantum communication and quantum cryptography, the question of how to determine the actual state of a quantum system has gained renewed interest. Recently, a large number of significant schemes for diagnosing the states of a system have been proposed based on the quantum tomography technique (i.e., reconstruction of the states by measuring enough observables, given many copies of a quantum state) [16]. However, for some solid state qubit systems, it is very difficult to implement quantum tomography since usually very few, if not just one, observables can be measured.

In this work, we propose a method for extracting the relative phase of a $n$-qubit two-component entangled state with the general form of

$$
\sqrt{p}\left|i_{1} i_{2} \ldots i_{n}\right\rangle+e^{i \varphi} \sqrt{1-p}\left|\bar{i}_{1} \bar{i}_{2} \ldots \bar{i}_{n}\right\rangle,
$$

(where $i_{l}, \bar{i}_{l} \in\{0,1\}, \bar{i}_{l}=1-i_{l}, \varphi$ is a relative phase, and $0<p<1$ ). The method presented here operates essentially by local Hadamard transformations and measurements along a single basis (the $z$ basis: $|i\rangle$ and $|\bar{i}\rangle$ ). This work is of significant interest because it provides a way to extract the relative phase from the state (1) and therefore can be used to determine an arbitrary multiqubit two-component entangled state. In addition, the extraction of the relative phase may have other applications in quantum information theory and high precision measurements $[17,18]$. In the following, we will further show how to distinguish the states (1) with arbitrary weighing factors (any entanglement degree) and relative phases from a class of multiqubit mixed states.

Before our presentation, let us give a brief discussion on the states (1). For $p=1 / 2$ and $n=2$, the states (1) are reduced to the well known Bell states; while for $p=1 / 2$ and $n>2$, they are called Greenberger-Horne-Zeilinger (GHZ) states [19]. The Bell or GHZ states play an important role in quantum information processing and communication and are of great interest in the foundations of quantum mechanics and measurement theory [1,20-22]. Recently, there has been much interest in generation and engineering of the Bell or GHZ states and several methods have been proposed for their generation in physical systems such as atoms, ions, quantum dots, charge qubits, and flux qubits [23-31]. On the other hand, for $0<p<1 / 2$, the states (1) are partially (non-maximally) entangled states, which may be caused by non-exact control of operations or decoherence. As is well known, a $n$-qubit maximally entangled state can be extracted from a set of partially entangled states with the same form of Eq. (1) by using entanglement purification protocol [32,33]. And, quantum communication (e.g., teleportation and cryptograph) based on partially entangled states of Eq. (1) have been discussed recently [34-36].

Now let us see how to extract the relative phase $\varphi$ of state (1). It can be shown that if a Hadamard transformation described by $|0\rangle \rightarrow \frac{1}{\sqrt{2}}(|0\rangle+|1\rangle)$ and $|1\rangle \rightarrow \frac{1}{\sqrt{2}}(|0\rangle-|1\rangle)$ is performed on each qubit, then the state (1) becomes 


$$
\begin{aligned}
& 2^{-n / 2}\left(\sqrt{p}+e^{i \varphi} \sqrt{1-p}\right) \sum_{\left\{x_{l}\right\}}\left( \pm\left|\left\{x_{l}\right\}\right\rangle\right) \\
& +2^{-n / 2}\left(\sqrt{p}-e^{i \varphi} \sqrt{1-p}\right) \sum_{\left\{y_{l}\right\}}\left( \pm\left|\left\{y_{l}\right\}\right\rangle\right) .
\end{aligned}
$$

Here, $\left|\left\{x_{l}\right\}\right\rangle=\left|x_{1} x_{2} \ldots x_{n}\right\rangle$ and $\left|\left\{y_{l}\right\}\right\rangle=\left|y_{1} y_{2} \ldots y_{n}\right\rangle$ are computational basis states of the $n$ qubits $\left(x_{l}, y_{l} \in\{0,1\} ; l=\right.$ $1,2, \ldots n)$, and $\sum_{\left\{x_{l}\right\}}\left( \pm\left|\left\{x_{l}\right\}\right\rangle\right)\left[\sum_{\left\{y_{l}\right\}}\left( \pm\left|\left\{y_{l}\right\}\right\rangle\right)\right]$ is a sum over all possible basis states $\left|\left\{x_{l}\right\}\right\rangle\left(\left|\left\{y_{l}\right\}\right\rangle\right)$ each containing an even (odd) number of "1"s. For instance, when $n=4, \sum_{\left\{x_{l}\right\}}\left( \pm\left|\left\{x_{l}\right\}\right\rangle\right)= \pm|0000\rangle \pm|1100\rangle \pm|1010\rangle \pm \cdots \pm|1111\rangle$. In Eq. (2), the \pm signs for each term $\left|\left\{x_{l}\right\}\right\rangle\left(\left|\left\{y_{l}\right\}\right\rangle\right)$ depend on the term $\left|i_{1} i_{2} \ldots i_{n}\right\rangle$ involved in the original state (1). That is, the choice for the + sign or the - sign is determined by the number of "1"s contained in the binary computational basis state $\left|i_{1} i_{2} \ldots i_{n}\right\rangle$ and the arrangement of " 1 " $\mathrm{s}$ in $\left|i_{1} i_{2} \ldots i_{n}\right\rangle$. It should be mentioned that the choice for the \pm signs does not affect the measurement results since all measurements throughout this paper are made on individual qubits along the $z$ basis.

Eq. (2) demonstrates that when the $n$ qubits were initially in the state (1), if a measurement in $z$-basis is made on each qubit after a Hadamard transformation on each qubit, the probability of finding an even number of qubits in the state $|1\rangle$ would be given by

$$
p_{\text {even }}=\frac{1}{2}+\sqrt{p(1-p)} \cos \varphi
$$

The result (3) shows that the relative phase $\varphi$ of the entangled state (1) can be determined using the procedure described above. Reasons for this are as follows. First, the value of $p$ can be obtained using a simple measurement in $z$-basis on each qubit alone, given many copies of the state (1). Second, the probability $p_{\text {even }}$ can be determined by measurements in $z$-basis on each qubit after performing a Hadamard transformation on each qubit. Therefore, one can determine the form of a multiqubit two-component entangled state (1), with the prior knowledge that the qubit system is prepared in a certain but unknown state belonging to a finite set of possible states (1). Finally, it is noted that the state (1) is reduced to a pure state of a single qubit when $n=1$. Hence, a pure state $\sqrt{p}|0\rangle+e^{i \varphi} \sqrt{1-p}|1\rangle$ of a single qubit with arbitrary $p$ and $\varphi$ can be confirmed using the above procedure.

In what follows our purpose is to show how to distinguish the entangled state (1) from a class of $n$-qubit mixed state:

$$
\rho_{\text {mix }}=p\left|i_{1} i_{2} \ldots i_{n}\right\rangle\left\langle i_{1} i_{2} \ldots i_{n}|+(1-p)| \bar{i}_{1} \bar{i}_{2} \ldots \bar{i}_{n}\right\rangle\left\langle\bar{i}_{1} \bar{i}_{2} \ldots \bar{i}_{n}\right| .
$$

Note that for the state (1) the probabilities of the $n$ qubits being in $\left|i_{1} i_{2} \ldots i_{n}\right\rangle$ and $\left|\bar{i}_{1} \bar{i}_{2} \ldots \bar{i}_{n}\right\rangle$ are given by $p$ and $1-p$, respectively. However, the same results are obtained when the $n$ qubits are in the mixed state (4). Hence, one needs to distinguish the state (1) from the mixed state (4) to make sure that the $n$ qubits are indeed prepared in the entangled state (1).

It is straightforward to show that if a Hadamard transformation is performed on each qubit, then the density operator (4) becomes

$$
\frac{p}{2^{n}} \sum_{\left\{x_{l}^{\prime}\right\}}\left( \pm\left|\left\{x_{l}^{\prime}\right\}\right\rangle\right) \otimes \sum_{\left\{x_{l}^{\prime}\right\}}\left( \pm\left\langle\left\{x_{l}^{\prime}\right\}\right|\right)+\frac{q}{2^{n}} \sum_{\left\{x_{l}^{\prime}\right\}}\left( \pm\left|\left\{y_{l}^{\prime}\right\}\right\rangle\right) \otimes \sum_{\left\{x_{l}^{\prime}\right\}}\left( \pm\left\langle\left\{y_{l}^{\prime}\right\}\right|\right) .
$$

Here, $\left|\left\{x_{l}^{\prime}\right\}\right\rangle=\left|x_{1}^{\prime} x_{2}^{\prime} \ldots x_{n}^{\prime}\right\rangle$ and $\left|\left\{y_{l}^{\prime}\right\}\right\rangle=\left|y_{1}^{\prime} y_{2}^{\prime} \ldots y_{n}^{\prime}\right\rangle$ are computational basis states of the $n$ qubits $\left(x_{l}^{\prime}, y_{l}^{\prime} \in\{0,1\} ; l=\right.$ $1,2, \ldots n)$, and $\sum_{\left\{x_{l}^{\prime}\right\}}\left( \pm\left|\left\{x_{l}^{\prime}\right\}\right\rangle\right)\left[\sum_{\left\{y_{l}^{\prime}\right\}}\left( \pm\left|\left\{y_{l}^{\prime}\right\}\right\rangle\right)\right]$ is a sum over all possible basis states. For instance, when $n=3$, $\sum_{\left\{x_{l}\right\}}\left( \pm\left|\left\{x_{l}^{\prime}\right\}\right\rangle\right)$ or $\sum_{\left\{y_{l}^{\prime}\right\}}\left( \pm\left|\left\{y_{l}^{\prime}\right\}\right\rangle\right)= \pm|000\rangle \pm|001\rangle \pm|010\rangle \pm|100\rangle \pm|011\rangle \pm|101\rangle \pm|110\rangle \pm|111\rangle$. The \pm signs for each term $\left|\left\{x_{l}^{\prime}\right\}\right\rangle\left(\left|\left\{y_{l}^{\prime}\right\}\right\rangle\right)$ are determined by the number of "1"s and the arrangement of "1"s in the basis state $\left|i_{1} i_{2} \ldots i_{n}\right\rangle$ involved in the density operator (4).

Eq. (5) shows clearly that for the mixed state (4), if each qubit is measured in $z$-basis after a Hadamard transformation on each qubit, the probability for an even number of qubits being found in the state $|1\rangle$ would be $1 / 2$. On the other hand, as shown above, for the $n$-qubit initial state (1), if the same operation is performed on each qubit, the probability of an even number of qubits being found in the state $|1\rangle$ is given by Eq. (3). Hence, in general, when $p_{\text {even }} \neq 1 / 2$, it can be concluded that the $n$ qubits are in the state (1).

However, for the special case of $\varphi= \pm \pi / 2$, the entangled state (1) also has $p_{\text {even }}=1 / 2$. Namely, the following $n$-qubit entangled state

$$
\sqrt{p}\left|i_{1} i_{2} \ldots i_{n}\right\rangle+e^{ \pm i \pi / 2} \sqrt{1-p}\left|\bar{i}_{1} \bar{i}_{2} \ldots \bar{i}_{n}\right\rangle
$$


could not be distinguished from the mixed state (4) using the procedures described above. However, we note that for this special case, the above method for entanglement verification based on single-basis measurement can still work. This is because, by performing a local phase shift operation on any one of the $n$ qubits, described by $|i\rangle \rightarrow|i\rangle,|\bar{i}\rangle \rightarrow$ $e^{i \pi / 2}|\bar{i}\rangle$, the state $(6)$ is transformed into

$$
\sqrt{p}\left|i_{1} i_{2} \ldots i_{n}\right\rangle \mp \sqrt{1-p}\left|\bar{i}_{1} \bar{i}_{2} \ldots \bar{i}_{n}\right\rangle
$$

i.e., the state (1) with $\varphi=\pi$ or 0 . The state (7) can obviously be distinguished from the mixed state (4) using the method described above because for them one has $p_{\text {even }}=\frac{1}{2} \mp \sqrt{p(1-p)} \neq 1 / 2$ from Eq. (2).

It should be noticed that the technique of distinguishing the entangled state (1) from the mixed state (4) by local operations and measurements on individual qubits was previously presented in the generation of entanglement of polarized photons (for details, see references [37-39]). However, we point out that in this work we considered a general case, i.e., the $n$-qubit entangled state of Eq. (1) with arbitrary $p$ and $\varphi(0<p<1)$. Namely, the state (1) considered here could be maximally-entangled states with $p=1 / 2$ and an arbitrary relative phase $\varphi$, or partially-entangled states with $0<p<1 / 2$ and an arbitrary relative phase $\varphi$. The results presented above are valid for any qubit system. Our work regarding the distinction between the generic state (1) and the mixed state (4) is a generalization of the previous work [37-39] to a multiqubit two-component entangled state with a general form, which is of great interest in both theory and experiment by itself.

A single-qubit Hadamard transformation can be easily achieved for many physical qubit systems. For examples, it can be done by locally rotating the polarization of a photon, by applying a $\pi / 2$ microwave pulse resonant with the transition between the two lowest levels (the two logical states of a qubit) $|0\rangle$ and $|1\rangle$ of a SQUID (superconducting quantum interference device) [31], or by applying a $\pi / 2$ two-photon Raman resonance pulse to the two lowest levels of an atom with a $\Lambda$-type three-level structure.

As a matter of fact, based on $|0\rangle=|+\rangle+|-\rangle$ and $|1\rangle=|+\rangle-|-\rangle$, it is noted that Hadamard transformations are not necessary for either extracting the relative phase $\varphi$ or verifying the entangled state (1), because the same results can be obtained by measuring each qubit in the $x$ basis $|+\rangle$ and $|-\rangle$, instead of a Hadamard transformation followed by a measurement in the $z$ basis $|0\rangle$ and $|1\rangle$. However, it should be mentioned that the single-qubit Hadamard transformations are needed for some important physical qubit systems, for which usually only one natural measurement basis ( the $z$ basis) is available. For instance, for superconducting charge (flux) qubits, which are promising candidate for scalable quantum information processing, it is very difficult to perform measurement in basis other than the charge (flux) degree of freedom.

In summary, we have shown that an arbitrary relative phase can be extracted from a multiqubit two-component entangled state based on local single-qubit Hadamard transformations and measurements along a single basis only. The method is relatively easy to be realized because there is no need for two-qubit operations and the qubits are allowed to be well separated in space. The present work provides a way to determine an arbitrary multiqubit twocomponent entangled state through the extraction of the relative phase, which is of great importance to quantum communication in general and quantum key distributions in particular. In addition, extracting the phase may have other applications in quantum information theory and high precision measurements. Finally, a detailed discussion on distinguishing the entangled state (1) (with an arbitrary entanglement degree and relative phase) from a class of multiqubit mixed states has been presented.

This work was partially supported by National Science Foundation (DMR-0325551), and by AFOSR, NSA, and ARDA through DURINT grant (F49620-01-1-0439).

[1] M. Hillery, V. Buzek, and A. Berthiaume, Phys. Rev. A 59, 1829 (1999).

[2] C. H. Bennett, G. Brassard, C. Crépeau, R. Jozsa, A. Peres, and W. K. Wootters, Phys. Rev. Lett. 70, 1895 (1993).

[3] M. Curty, M. Lewenstein, and N. Lütkenhaus, Phys. Rev. Lett. 92, 217903 (2004).

[4] C. H. Bennett, D. P. DiVincenzo, J. A. Smolin, and W. K. Wootters, Phys. Rev. A 54, 3824 (1996); W. K. Wootters, Phys. Rev. Lett. 80, 2245 (1998).

[5] C. H. Bennett, G. Brassard, S. Popescu, B. Schumacher, J. Smolin, and W. K. Wootters, Phys. Rev. Lett. 76, 722 (1996).

[6] V. Vedral, M. B. Plenio, K. Jacobs, and P. L. Knight, Phys. Rev. A 56, 4452 (1997).

[7] K. Zyczkowski, P. Horodecki, A. Sanpera, and M. Lewenstein, Phys. Rev. A 58, 883 (1998).

[8] T. C. Wei and P. M. Goldbart, Phys. Rev. A 68, 042307 (2003).

[9] P. Horodecki and A. Ekert, quant-ph/0111064; P. Horodecki, quant-ph/0111082. 
[10] Y. C. Eldar, Phys. Rev. A 67, 042309 (2003).

[11] O. Gühne et al., Phys. Rev. A 66, 062305 (2002).

[12] P. Hyllus, C. Moura Alves, D. Bruß, and C. Macchiavello, quant-ph/0405164.

[13] G. Tóth and O. Gühne, quant-ph/0405165.

[14] M. Bourennane et al., Phys. Rev. Lett. 92, 087902 (2004).

[15] B. Schelpe, A. Kent, W. Munro, and T. Spiller, Phys. Rev. A 67, 052316 (2003).

[16] D. F. V. James et al., Phys. Rev. A 64, 052312 (2001); R. T. Thew et al., ibid. 66, 012303 (2002); R. Das et al., ibid. 67, 062304 (2003); D. Cavalcanti et al., ibid. 71, 014301 (2005); Z. Hradil, ibid 55, R1561 (1997); Z. Hradil et al., ibid 62, 014101 (2000); J. Rehacek et al., ibid. 63, 040303 (2001); K. Banaszek et al., ibid. 61, 010304 (1999); U. Leonhardt, Measuring the Quantum State of Light (Cambridge University Press, Cambridge, 1997).

[17] J. J. Bollinger, W. M. Itano, D. Wineland, and D. Heinzen, Phys. Rev. A 54, 4649 (1996).

[18] C. A. Sackett, D. Kielpinski, B. E. King, C. Langer, V. Meyer, C. J. Myatt, M. Rowe, Q. A. Turchette, W. M. Itano, D. J. Wineland, and C. Monroe, Nature (London) 404, 256 (2000).

[19] D. M. Greenberger, M. A. Horne, and A. Zeilinger, Phys. Today 46(8), 22 (1993), and references therein.

[20] See, for many references, S. Bose, V. Vedral, and P. L. Knight, Phys. Rev. A 57, 822 (1998).

[21] D. P. DiVincenzo and P. W. Shor, Phys. Rev. Lett. 77, 3260 (1996).

[22] J. Bollinger, W. M. Itano, D. J. Wineland, and D. J. Heinzen, Phys. Rev. A 54, R4649 (1996).

[23] L. M. Duan and H. J. Kimble, Phys. Rev. Lett. 90, 253601 (2003).

[24] J. I. Cirac and P. Zoller, Phys. Rev. A 50, 2799 (1994).

[25] S. B. Zheng, Phys. Rev. Lett. 87, 230404 (2001).

[26] J. Steinbach and C. C. Gerry, Phys. Rev. Lett. 81, 5528 (1998).

[27] K. Mølmer and A. Sørensen, Phys. Rev. Lett. 82, 1835 (1999).

[28] X. Wang, M. Feng, and B. C. Sanders, Phys. Rev. A 67, 022302 (2003).

[29] S. L. Zhu, Z. D. Wang, and P. Zanardi, e-print, quant-ph/0403004.

[30] C. P. Yang, S. I. Chu, and S. Han, Phys. Rev. A 67, 042311 (2003).

[31] C. P. Yang and S. Han, Phys. Rev. A 70, 062323 (2004).

[32] S. Bose1, V. Vedral, and P. L. Knight, Phys. Rev. A 60, 194 (1999).

[33] W. Dür, H. Aschauer, and H.-J. Briegel, Phys. Rev. Lett. 91, 107903 (2003); H. Aschauer, W. Dür, and H.-J. Briegel, Phys. Rev. A 71, 012319 (2005).

[34] Wan. L. Li, C. F. Li, and G. C. Guo, Phys. Rev. A 61, 034301 (2000).

[35] H. Kim, Y. W. Cheong, and H. W. Lee, quant-ph/0404172.

[36] M. Koashi and N. Imoto, Phys. Rev. Lett. 79, 2383 (1997).

[37] P. G. Kwiat, K. Mattle, H. Weinfurter, A. Zeilinger, A. V. Sergienko, and Y. Shih, Phys. Rev. Lett. 75, 4337 (1995).

[38] D. Bouwmeester, J. W. Pan, M. Daniell, H. Weinfurter, and A. Zeilinger, Phys. Rev. Lett. 82, 1345 (1999).

[39] Jian-Wei Pan, Matthew Daniell, Sara Gasparoni, Gregor Weihs, Anton Zeilinger, Phys. Rev. Lett. 86, 4435 (2001). 